\title{
Will Intelligent Vehicles Evolve into Human-peer Robots?
}

\author{
Mauro Da Lio, Member, IEEE, Francesco Biral, Marco Galvani, and Andrea Saroldi.
}

\begin{abstract}
This paper aims at stimulating the discussion on the future of Intelligent Vehicles. It is a position paper, indicating converging technologies that, in our opinion, will have to be used in future Intelligent Vehicles. We present a vision according to which Intelligent Vehicles will evolve into Human-peer Robots, here called Co-Drivers. Co-Drivers will be able to "understand" human drivers and to form symbiotic systems with them. The general architecture of Co-Drivers, the building blocks and the technologies that are needed to bring them to life are discussed, pointing out which parts have been already researched and which gaps still remain. We clarify what "understanding driver" actually means and how a joint system can be obtained. The paper will identify research needs and paths, and hopefully trigger interest.
\end{abstract}

\section{INTRODUCTION. THE LOST "HORSE"?}

$\mathrm{M}$ ANKIND used animals, and especially horses, as transportation systems for thousand years. Last century they were eventually replaced by motor vehicles because of range, speed, capacity and costs. However, something was lost: the intelligence of the animals was traded with power. In a recent book [1], Norman recalls the interaction between a rider and a horse as one example of how future intelligent things should work:

Think of skilled horseback riders. The rider "reads" the horse, just as the horse can read its rider. (...). This interaction (...) is of special interest because it is an example of two sentient systems, horse and rider, both intelligent, both interpreting the world and communicating their interpretations to each other.

According to Norman, here the key point is the peer-topeer cooperation of two "sentient" systems. In this example, a man and an animal, but the animal stands for a future intelligent artificial system.

Norman was not the only one to point out such a smart collaboration. The H-metaphor (i.e., rider-horse metaphor) was also proposed by Flemish, originally in the aerospace

Manuscript received January 15, 2012. This work was supported in part by the European Commission under Grants FP6 507075 (PReVENT), FP7 216355 (SAFERIDER), FP7 246587 (interactIVe).

Mauro Da Lio is with the Department of Mechanical and Structural Engineering, University of Trento, Italy (corresponding author, phone: +39 0461282504, fax: +39 0461282599, email: mauro.dalio@unitn.it).

Francesco Biral is with the Department of Mechanical and Structural Engineering, University of Trento, Italy (email: francesco.biral@unitn.it).

Marco Galvani is with the Department of Mechanical and Structural Engineering, University of Trento, Italy (email: marco.galvani@unitn.it).

Andrea Saroldi is with Centro Ricerche Fiat (CRF), Orbassano, TO, Italy (email: andrea.saroldi@crf.it). domain, as a guideline for interactions between vehicle and driver [2]. More in general, prospecting for smart collaboration between humans and vehicles has been described by other authors too, such as Heide [3] and Inagaki [4].

In an even broader sense, the envisioned cooperation falls into the category of human-robot interactions [5], [6], and in particular in that kind of interaction where the robot behaves as a peer or tutor or mentor or companion (etc.).

The sharing of authority and the mixed initiative are two crucial aspects of peer cooperation. Adaptive Automation [7], [8] refers to systems in which both the user and the system can initiate changes in the level of automation, producing modes of automation more closely tied to operator needs at any given moment.

\section{PATHS TOWARDS HUMAN-PEER CO-DRIVERS}

Let us call "Co-Driver" a robotic artificial system that implements the vision described by Norman, i.e., a robot that is smart enough to "understand" the driver and to form with the driver a symbiotic system. Of course this includes the ability of the robot to adapt the level of automation (e.g., from supervision to nearly fully autonomous drive), but the robot is much more. The H-metaphor helps us to understand how this system could work, and guides us to the development (including understanding possible issues), but does not tell anything about how this robot has to be engineered.

In the following, we present ideas that we matured in many years working on the design of Advanced Driver Assistance Systems, including three European projects (PReVENT, SAFERIDER and interactIVe). We introduce the general architecture of co-drivers and discuss building blocks that in part have already been developed in many projects (including, but not limited to, our own). We discuss the technologies that can be used to build co-drivers, and especially cognitive systems versus systems engineered by design, and point out maturity gaps and research needs.

The paper, by its nature, will not provide conclusive solutions. It is rather intended to stimulate discussion and suggest research paths.

\section{ARCHITECTURES}

\section{A. What Co-Drivers may be.}

There are several terms that can be used to refer to CoDrivers. None of them gives the full picture, but recalling these terms is useful to point out the main aspects that form a Co-Driver, and link Co-Drivers to the State of the Art. 
Co-Pilot is a term borrowed from the aviation domain. Strictly speaking it indicates the second pilot of an aircraft. In a broader sense it may also indicate an automatism (more properly referred as Autopilot) that can take care of some guidance tasks. Using "co-pilot" to indicate an artificial second pilot casts some extra light on the H-metaphor.

An autopilot can be turned on and off on demand, or may provide intermediate, dynamically enabled, levels of automation taking care of some tasks and leaving others to the driver.

As an example, the project HAVEit [9] had, among its objectives, the study of a joint system formed by a driver and a simple artificial co-pilot (see below under "virtual driver" for further discussion about co-pilots). In particular HAVEit focused on dynamic allocation of tasks between the driver and co-pilot and on transitions between levels of automation.

Virtual driver, is a special case of "virtual users" or "digital human beings". These are models of human beings that can serve various design and simulation purposes [10]. In our case a virtual driver is a comprehensive driver model, which can be used to predict how a real human would drive in a certain situation. In facts, the robot (as was the horse in the metaphor) must possess an internal model of human motion patterns. Below we will discuss the main requirements and features that such a model must possess.

As an example, in PReVENT project we used a virtual driver to generate a "reference maneuver" [11] to be compared with the actual behavior of the real driver. Mismatch between real driver and virtual driver was the main method to assess the situation and derive warnings.

Another example is the project DIPLECS [12], which demonstrated an artificial cognitive system learning to interpret the intentional behavior of a real driver.

Companion Driver. It may indicate a special class of companion robots; that is: a friendly artificial companion that assists the human (especially for people with limited skills and elders). Emphatic links is stressed in companion robotics, and in our case means a deep mutual understanding, which can help making the co-driver accepted by the human. A "Trip Mate" concept has been presented in [13].

Driver tutor is a self-explanatory term. Some Driver Assistance Systems works like discrete tutors.

\section{B. Co-Drivers architectures.}

Fig1. Introduces the main elements of a symbiotic diver co-driver system.

What the figure shows is that there are two "sentient" entities living on each other side. For the moment we leave the driver and co-driver blocks empty. However, since we know that the co-driver will have a user model inside, it is indicated with the nested block named "virtual driver". The dashed arrow shows possible communication between the two, in- cluding observation of driver preparatory movements [40].

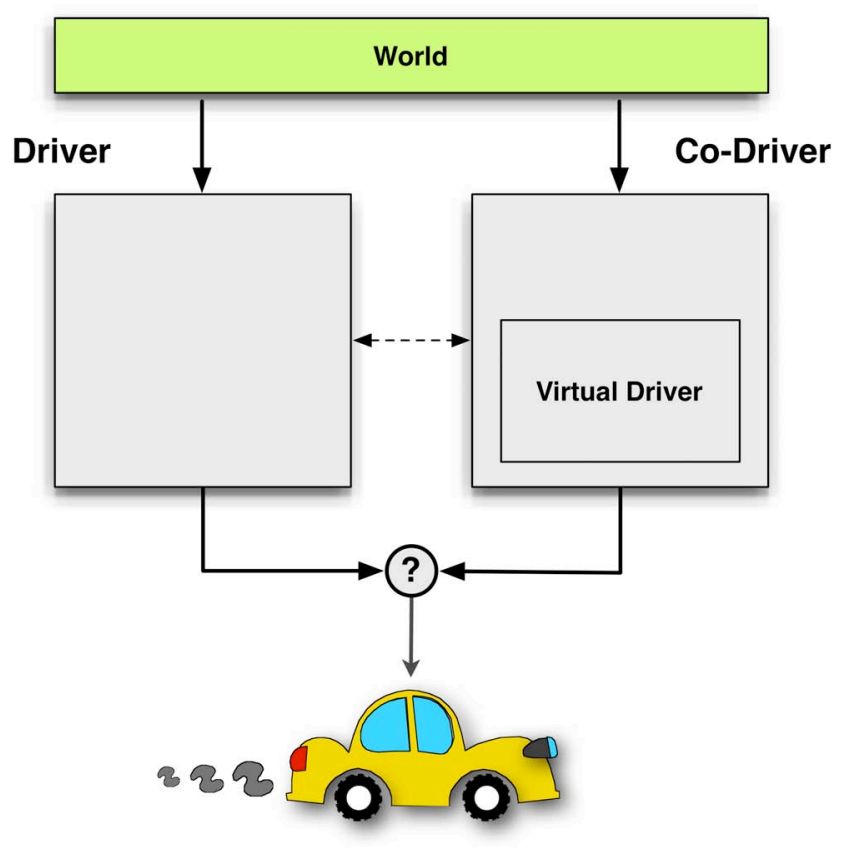

Fig. 1. A symbiotic Driver and Co-Driver system is formed by two sentient systems that interprets the world with their own perception, exchange each other information, and ultimately fuse their control action.

Fig.1 is useful to spot two first points:

1) The perception issue. Both driver and co-driver sense the world, but they have different senses and different overall perception, which might end up into mismatching representations of the world. Without dissecting all the differences (and technologies) of the two perception systems, the important thing is that the two entities might be forced to take decisions on disagreeing representations of the world.

One thing that must be stressed is that there is no way to recover incorrect, missing or insufficient information in perception. Machine perception must be reliable and complete.

Reliability of machine perception system is thus a prerequisite for Co-Drivers. In our opinion this requirement is not completely achieved yet (at least for Co-Drivers).

2) The authority issue. Somewhere driver and co-driver must be "fused" together to jointly control the vehicle, which is shown in Fig.1 with the question mark. Joint control means that driver and co-driver behave like a unity, which may be somewhat different than the split control that can be achieved by certain autopilot solutions.

Joint control can be obtained by revising the above architecture, but first let us have a look at what may be inside driver and co-driver blocks.

\section{CO-Driver Purpose AND ARCHITECTURES REVISITED}

Consider the example situation of Fig. 2 and ask how a joint system should work. In other words, how can we make a smart, green, safe (etc.) joint system?

Perhaps the best way to answer this question is to answer 
first another question:

How would a human driver, expert, attentive, capable, green aware, knowledgeable (etc.), drive?

This question introduces the idea that a Co-Driver system can be built around a properly made virtual user model, which explains why the Co-Driver block in Fig.1 includes a nested "virtual driver".

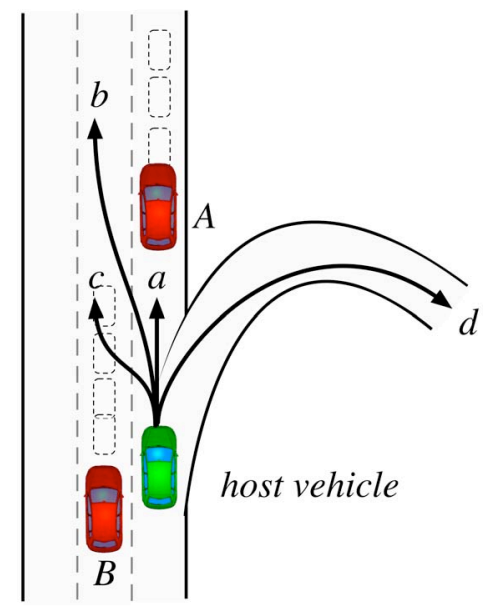

Fig. 2. Multiple ways of driving arise from multiplicity of goals and introduces a hierarchy in driver models.

Fig. 2 shows that there may be multiple answers to the above question: the way an expert human drives depends on what is his/her goal. In the example, there are 4 possible alternative short-term (tactical) goals. For any goal, there is a different way to drive.

This in turn introduces the concept that user models are hierarchical, with higher levels of the hierarchy being in charge of setting goals and lower levels of developing tactics and detailed control. Levels further higher could exist too, and represent psychological states and long-term strategies, which affect the way shorter-term goals are set (e.g., the decision whether to follow $a$ or overtake $b$ may stem from time pressure).

Thus, the first feature to be considered for a virtual driver model is the existence of several concurrent control, planning, sensing and cognition tasks, organized in a hierarchy, where slow cognitive tasks occur at the top and faster control loops at the bottom.

The Michon model [14], in mid ' 80 , describes three layers, referred as strategic, tactical (or maneuvering) and operational (or control) levels that occur in different time frames, from tens/hundreds of seconds to a fraction of second. Following Michon, other psychological studies were proposed. For example, Hatakka et. al. [15] Introduced a fourth level on top, which deals with psychological states and was referred as "goals for life and skills for living".

More recently Hollnagel introduced the Extended Control Model (ECOM) [16], [17], with four concurrent interacting layers called tracking, regulating, monitoring and targeting (Fig.3).

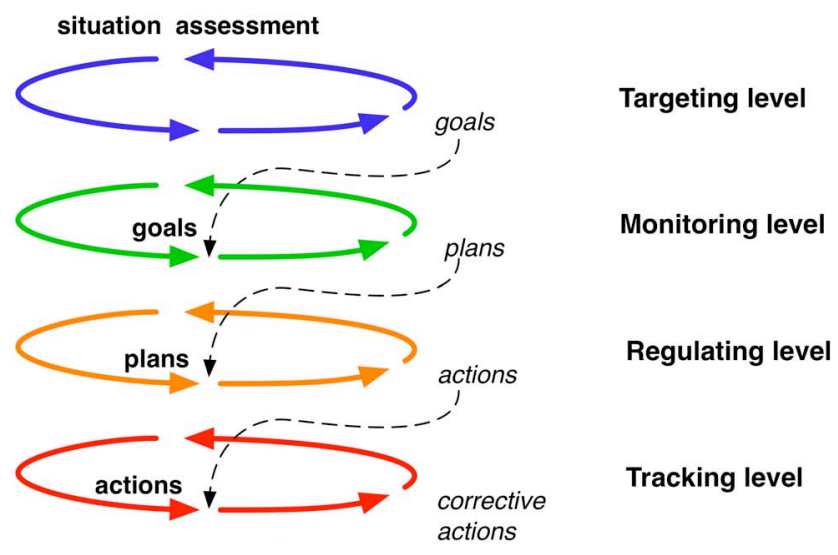

Fig. 3. Hollagel's Extended Control Model (ECOM)

The ECOM explains how the different levels interact. Namely the ECOM is a subsumptive architecture [18], meaning that every loop can activate, suspend and deactivate different alternative sub-loops (although Fig.1 does not show, there can be many alternative sub-loops). In addition every higher loop sets the goals for the active sub-loop.

For example, in Fig.2 the Monitoring level may set the goal (e.g., overtake $b$ ) and the regulating level will produce the trajectory.

Hollnagel does not make any claim that the four loops actually exist and occur in the human mind in that form. Rather they satisfy a tradeoff between modeling needs and complexity, and well reflect the diversity of time frames in which vehicle control takes place. From bottom to top: the tracking level is related to minute chassis control and disturbance rejection; the regulating level is related to vehicle maneuvering (producing space-time trajectories); the monitoring level is related to setting short-term goals (e.g., overtake), which can arise from keeping track of the progress towards final destination, signs and indications, traffic conditions etc.; the targeting level means long-term goals like route and driving styles (e.g., go home quickly).

\section{A. Artificial Cognitive Systems approach}

The ECOM architecture has been embodied in project DIPLECS [12], except for the highest targeting level loop (where to go).

DIPLECS used an Artificial Cognitive System (ACS) to model ECOM loops as Perception-Action (PA) loops [19][21]. This approach is quite promising: Perception-Action loops can be learnt from examples of real drivers. Perception-action approach makes a minimal assumption as regards the driver's perceptual system, and is thus less prone to generalization error from small numbers of training instances. Once learnt, PA maps provide the means to translate perceptual goals into actions to achieve that goal. For example, suppose that the monitoring level sets the goal " $b$ " (overtake in Fig.2) to the regulating level. At this level the goal is then 
translated into actions (space-time trajectory), by the trained PA map, which then feeds the tracking level.

The virtual driver made in this way will "emulate" the real driver.

In principle, one advantage of ACS approach is that the system can evolve, thus overcoming the many "hard-topredict" and "hard-to-pre-code" issues of traditional preengineered approaches. Another advantage is that perception itself is part of the cognitive process. Thus, learnt perception can help addressing also the perception issue above mentioned.

\section{B. Receding horizon optimal control approach}

One different approach to map short-term goals (Fig.2: $a$, $b, c, d$ ) into space-time trajectories is Optimal Control (OC).

Optimal Control has been successfully used to explain the trajectories that humans produce in general contexts [22][23], and in driving [24]-[26], including our contributions [27], [11]. A receding horizon scheme means that plans are repeatedly updated during the execution of the movement itself [11], [23]. With receding horizon, if a deviation is detected, a new plan is developed to achieve the original target from the new state. There is no return to the previously computed trajectory but a new trajectory developing from the deviated position is produced. This has been stated as minimal intervention principle, which states that taskirrelevant deviations are left uncorrected [23]. In a concurrent subsumptive architecture like in Fig.3, receding horizon means that a loop (say the regulating loop) continues to target the set goal, adjusting its own deviations, until a new goal is set by the higher loop.

The following key ideas are emphasized in OC:

\section{1) Modeling human motor strategies}

A number of studies have been carried out to understand which may be the criteria that produce human motion in general [22], [23], [28]-[31], and for the specific domain of driving [32]-[39].

Minimum jerk is one of the most important movement rules. We can regard minimum jerk as a principle of "laziness" or maximum "smoothness". For example, in driving, minimum jerk trajectories are those that minimize the root, mean square (rms) value of driver control.

However, minimum jerk is not the only criterion that stands at the origin of human motor strategies. Often motor strategies arise from a tradeoff of several other criteria (e.g., time, energy, thresholds and saturations, etc.) [11], [23], [39]. In such cases a composite cost function, weighting different criteria together, allows to model a variety of motor strategies. For example, a cost function, combining minimum jerk and minimum time, models a variety of human behaviors under different time pressure.

In a subsumptive architecture (Fig.3), many optimal control problems, based on different cost functions or parameterized cost functions, may be turned on and off by the immediately upper level, to model, for example, different problems and different driving styles.

\section{2) Driver mental model of the controlled vehicle}

Several studies [25]-[26] pointed out that drivers have an internal model of the dynamics of the vehicle they are controlling, and that they manipulate this model to foresee the effects of their actions (also referred as driver having preview abilities).

Since optimal control is a method to "steer" a dynamic system to a desired state, a model of the dynamic system has to be supplied as an ingredient [11]. When optimal control is used to model a driver trajectory-planning activity, this means that the supplied model of the dynamic system represents the mental model of the driver, and that the methods represent the manipulation of the model to achieve the set goal.

The dynamic model, in pre-engineered systems, has always been defined by the designer (e.g., [11]). However, it could be also provided in the form of a learnt PA map by an artificial cognitive system, thus modeling a driver who evolves it own knowledge of the dynamics of the system.

\section{Towards human-peer robots}

We are now in condition to put things together and clarify how a co-driver may "understand" the driver and how driver and co-driver may form a unity.

To clarify what the term "understand" means, let us first resort to metaphors and think the Co-Driver were a human being (e.g., a driving tutor). The movement of humans is predictable to other human beings because, as explained above, human motor strategies are wired in the human brain and people are naturally aware of these motion schemes. By watching how other human beings move, people can quickly "reverse engineer" their knowledge about human motor strategies and identify the goal. The key element is establishing a link between some observed initial motor activity and a meaningful goal. The same happens for a horse in the $\mathrm{H}$ metaphor, who links signs from the rider to meaningful goals.

Out of the metaphor, a Co-Driver, which is made knowledgeable of human motor strategies (because including a virtual driver made with one or more of the technologies discussed so far), would also be able to map driver activities onto meaningful goals. In a word a Co-Driver grounds (assigns a meaning) driver intentions. A vast amount of literature exists about forecasting driver behavior/intentions [40]. However, here a new step is taken: in addition to forecasting future driver actions a Co-Driver grounds these actions to a goal, a motivation (or hopefully a psychological state).

Fig. 4 provides a revised architecture. Now the driver and co-driver blocks are modeled with an ECOM. The ECOM states of the driver (i.e., the goals at all levels) are not directly observable, but the comparison of driver and co-driver outputs (plus any additional information exchange) provides means to estimate driver ECOM states. In a broad sense this is a state estimation problem, where a model of a system is updated to match its output.

Understanding the driver means that driver ECOM states, i.e., driver goals at all levels are estimated. The topmost lev- 
el (targeting level) makes a partial exception: where the driver has long-term goals, such co-driver long-term goals do not exist (in the sense that the co-driver has no aim to go anywhere). However, long-term goals of the co-driver may be to "second" the driver (i.e., the topmost aim of the codriver might be "seconding" the driver, which opens a whole set of interesting perspectives, far beyond what can be discussed in this paper).

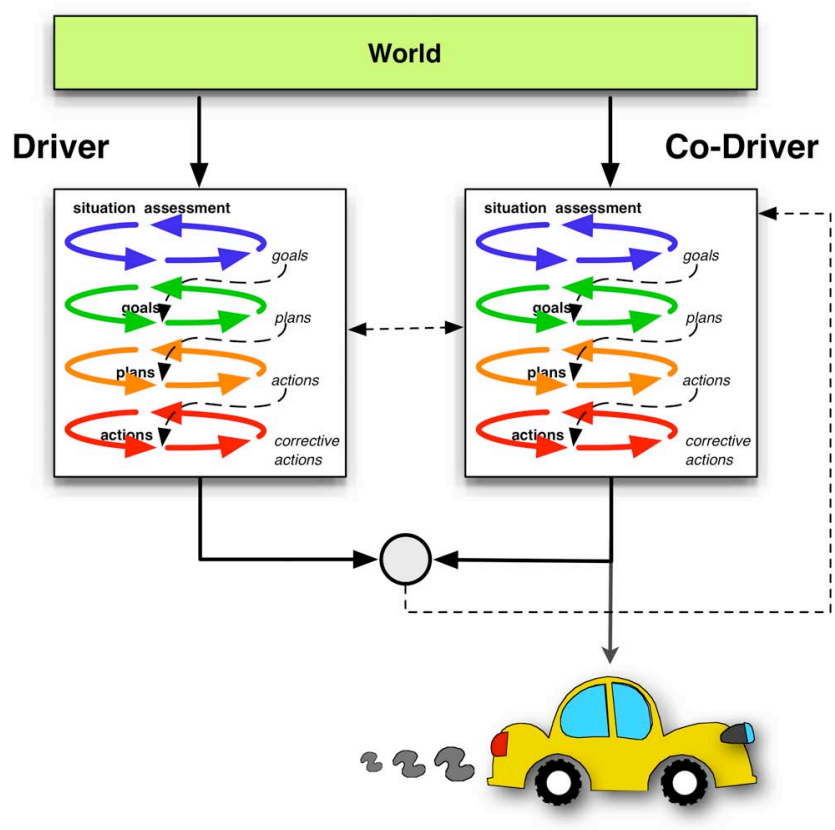

Fig. 4. Revised joint system architecture.

The last thing Fig.4 shows is that vehicle is actually controlled by the co-driver. Nonetheless the Co-Driver will almost always move according to estimated driver goals (maintaining safety and fault-tolerant behavior if the perception system of the co-driver is sufficiently intelligent). Hints from the driver may identify meaningful goals, which the co-driver will pursue, just like hints to a horse are sufficient to make the horse find a goal and go there. Should the driver and co-driver diverge, hints from the driver will make the co-driver realize that a new goal has to be searched. The codriver (provided that the perception issue is solved) could also spot inconsistent erroneous driver requests, including driver maneuvers that have no clear goal or match clearly mistaken goals, and, only in this case, the co-driver could adopt a different and safer behavior.

\section{CURRENT ONGOING IMPLEMENTATION}

We mentioned that building blocks have already been tested in some projects. Today, the interactIVe project [41] is making, among the others, a simple Co-Driver implementation, as shown in Fig.5. This is for the Continuous Support (CS) function in subproject 4, which aims at providing preventive early warning and advices in a holistic fashion, integrating the coverage of several types of risks.

The interactIVe Co-Driver implements the ECOM regu- lating and monitoring levels, respectively called Maneuvers and Goals in Fig.5. The tracking level is not needed because no active intervention is foreseen for CS function.

The regulating level is implemented with a family of parametric Receding Horizon Optimal Control problems. Each addresses a parametric different type of possible goal ranging from green driving, in-lane driving, driving on lane extensions, lane change and overtake and obstacle management.

The monitoring level is implemented via a Finite State Machine, which turns on and of the parameterized OC problems.

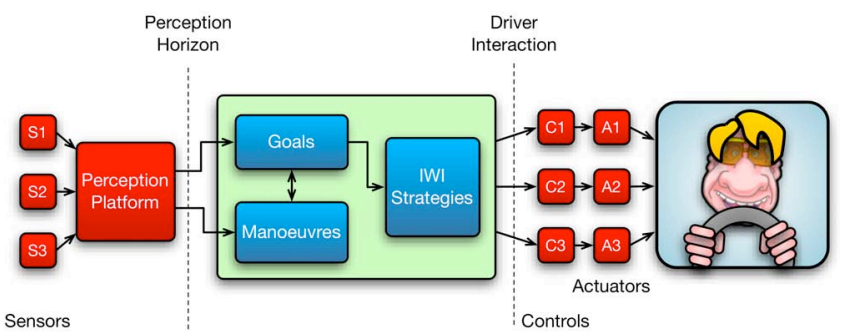

Fig. 5. Co-Driver for Continuous Support function in interactIVe project.

At any time the Co-Driver evaluates a couple of possible maneuvers: the one which looks better matching driver behavior (spotting any problem), and the one the Co-Driver would recommend (e.g. a safer or greener alternative). Evaluated maneuvers are then passed to the human machine interaction manager (I.W.I. Strategies).

\section{CONCLUSION}

This paper introduced the idea of Co-Drivers behaving as Human-peer robots and forming joint systems with drivers.

A general architecture for a joint system has been proposed and discussed.

A first key element of this architecture is that Co-Drivers must be aware of human motor strategies, which in turn calls for embedding a virtual user and for accurate modeling of human motor strategies.

A second element involves the concept of "understanding the driver" which can be traced down to a problem of identification or state estimation. The driver is modeled with a subsumptive ECOM, and ECOM states are identified comparing driver and co-driver output (which in turn stresses the good quality of virtual user models).

Technologies that have been used to make some building blocks have been pointed out. Artificial Cognitive Systems, namely based on Perception-Action schemata, has been used to emulate virtual users in DIPLECS project. Receding horizon optimal control has also been used to model virtual users for ADAS applications in PReVENT, SAFERIDER and is being used today in the interactIVe project to build a twolayer Co-Driver.

The next steps are integrating various technologies (PA, $\mathrm{OC}$, etc.) to form an effective virtual driver; researching the ECOM state estimation (its quality) and the joint system. 


\section{ACKNOWLEDGMENT}

This work was also supported by the European Commission under interactIVe, a large scale integrated project part of the FP7-ICT for Safety and Energy Efficiency in Mobility.

The authors would like to thank all partners within interactIVe for their cooperation and valuable contribution.

\section{REFERENCES}

[1] D. Norman, The Design of Future Things. MIT Press, 2007 (p. 19).

[2] F.O. Flemish, C. A. Adams, S. R. Conway, K. H. Goodrich, M. T. Palmer, P. C. Schutte, "The H- Metaphor as a guideline for vehicle automation and interaction," NASA/TM-2003-212672, NASA Langley Research Center, Hampton, VA, USA, 2003.

[3] A. Heide, K. Henning, "The "cognitive car": A roadmap for research issues in the automotive sector," Annual Reviews in Control, vol. 30, pp. 197-203, 2006.

[4] T. Inagaki, "Smart collaboration between humans and machines based on mutual understanding," Annual Reviews in Control, vol. 32, pp. 253-261, 2008.

[5] M. A. Goodrich, A. C. Schultz, "Human-Robot Interaction: A Survey: Foundations and Trends", Human-Computer Interaction, vol. 1 (3), 2007.

[6] S. Thrun, "Toward a Framework for Human-Robot Interaction", $\mathrm{Hu}$ man-Computer Interaction, vol. 19 (1-2), pp. 9-24, 2004.

[7] R. Parasuraman, T. B. Sheridan, "A Model for Types and Levels of Human Interaction with Automation", IEEE Trans. on systems, man, and cybernetics - Part A: Systems and humans, vol. 30 (3), May 2000.

[8] M. W. Scerbo, "Theoretical perspectives on adaptive automation", in Human performance in automated systems: Theory and applications, R. Parasuraman and M. Mouloua, Ed., Mahwah, NJ: Lawrence Erlbaum Associates, 1996.

[9] [Online]. Available: http://www.haveit-eu.org/

[10] V. G. Duffy (Ed.), "Digital Human Modeling," Proc. 2nd Int. Conf., ICDHM 2009, San Diego, CA, USA, July 19-24, 2009

[11] E. Bertolazzi, F. Biral, M. Da Lio, A. Saroldi, F. Tango, "Supporting drivers in keeping safe speed and safe distance: The SASPENCE subproject within the European framework programme 6 integrating project PReVENT," IEEE Trans. on Int. Trans. Sys., vol. 11 (3), pp. 525538,2010 .

[12] [Online]. Available: http://www.diplecs.eu/

[13] D. Scapaticci, U. Fermi, A. Saroldi, "Safety and Comfort Driver Support Functions in Trip Mate Concept Car," in Proc. 7th World Congress on Intelligent Transport Systems, Torino, 2000.

[14] J. A. Michon, "A critical view of driver behavior models: what do we know, what should we do?," in Human behavior and traffic safety, L. Evans and R.C. Schwing (Ed.), New York: Plenum Press, 1985, pp. 485-524.

[15] M. Hatakka, E. Keskinen, N. P. Gregersen, A. Glad, K. Hernetkoski, "From control of the vehicle to personal self-control; broadening the perspectives to driver education," Transportation Research Part F: Traffic Psychology and Behaviour, vol. 5-3, pp. 201-215, 2002.

[16] E. Hollnagel, D. D.Woods, "Cognitive systems engineering: new wine in new bottles," Int. J. Hum.-Comput. Stud., vol. 51 (2), pp. 339-356, 1999.

[17] E. Hollnagel, D. D. Woods, Joint Cognitive Systems: Foundations of Cognitive Systems Engineering. Boca Raton, FL: CRC Press, Taylor \& Francis Group, 2005, pp. 149-154.

[18] R. A. Brooks, "A robust layered control system for a mobile robot," IEEE Journal of Robotics and Automation, vol. 14 (23), 1986.

[19] A. H. Wechsler, "Integrating perception action and learning," SIGART Bull., vol. 2 (4), pp. 25-28, 1991.

[20] G.H. Granlund, "An associative perception-action structure using a localized space variant information representation," in Proceedings of Algebraic Frames for the Perception-Action Cycle (AFPAC), Kiel, Germany, 2000.

[21] M. Felsberg, J. Wiklund, G. Granlund, "Exploratory learning structures in artificial cognitive systems," Image and Vision Computing, vol. 27 (11), pp. 1671-1687, 2009.
[22] P. Viviani, T. Flash, "Minimum-jerk, two-thirds power law, and isochrony: converging approaches to movement planning," J. Exp. Psychol., vol. 21, pp. 32-53, 1995.

[23] D. Liu, E. Todorov, "Evidence for the Flexible Sensorimotor Strategies Predicted by Optimal Feedback Control," Journal of Neuroscience, vol. 27 (35), pp. $9354-9368,2007$.

[24] G. Prokop, "Modeling Human Vehicle Driving by Model Predictive Online Optimization", Veh. Sys. Dyn., vol. 35 (1), pp. 19-53, 2001.

[25] C. C. Macadam, "Understanding and Modeling the Human Driver," Veh. Sys. Dyn., vol. 40 (1), pp. 101-134, 2003.

[26] M. Plöchl, J. Edelmann, "Driver models in automobile dynamics application," Veh. Sys. Dyn., vol. 45 (7), pp. 699 -741, 2007.

[27] V. Cossalter, M. Da Lio, R. Lot, L. Fabbri, "A general method for the evaluation of vehicle maneuverability with special emphasis on motorcycles," Veh. Syst. Dyn., vol. 31, pp. 113-135, 1999.

[28] M. Breteler, R. Meulenbroek, S. Gielen, "An evaluation of the minimum-jerk and minimum torque-change principles at the path, trajectory, and movement-cost levels," Motor Control, vol. 6, pp. 69-83, 2002.

[29] G. Arechavaleta, J. Laumond, H. Hicheur, A. Berthoz, "An optimality principle governing human walking," IEEE Transactions On Robotics, vol. 24, pp. 5-14, 2008.

[30] K. Abdel-Malek, Z. Mi, J. Yang, K. Nebel, "Optimization-based trajectory planning of the human upper body," Robotica, vol. 24, pp. 683-696, 2006.

[31] Q. Pham, H. Hicheur, G. Arechavaleta, J. Laumond, A. Berthoz, "The formation of trajectories during goal-oriented locomotion in humans. II. A maximum smoothness model," European Journal of Neuroscience, vol. 26, pp. 2391-2403, 2007.

[32] R.S., Rice. "Measuring car-driver interaction with the g-g diagram," SAE Paper 730018, 1973.

[33] Y Hisaoka, M. Yamamoto, A. Okada, "Closed-loop analysis of vehicle behavior during braking in a turn", JSAE Review, vol. 20, pp. 537 542, Apr 1999.

[34] W. Mitchell, M. Unger, R. Scroer, D. Grisez, "Training test drivers with data acquisition", SAE Technical Paper Series, 2000-01-3568.

[35] W. Bartlett, L. L. C. Oren Masory, B. Wright, "Driver Abilities in Closed Course Testing", SAE Technical Paper Series, 2000-01-0179.

[36] S. T., Godley, "A driving simulator investigation of perceptual countermeasures to speeding", Ph.D. Thesis, Department of Psychology Monash University, 1999.

[37] M. Lappe, A. Grigo, F. Bremmer, H. Frenz, R. J. V. Bertin, I. Israel, "Perception of Heading and Driving Distance From Optic Flow", in Proc. Driving Simulation Conference 2000, Paris, 2000.

[38] W. Chee and M. Tomizuka, "Lane change maneuver of automobiles for the intelligent vehicle and highway system (IVHS)," in Proc. Amer. Control Conf., 1994, pp. 3586-3587.

[39] F. Biral, M. Da Lio, and E. Bertolazzi, "Combining safety margins and user preferences into a driving criterion for optimal control-based computation of reference maneuvers for an ADAS of the next generation," in Proc. IEEE Intell. Veh. Symp., Las Vegas, NV, pp. 36-41, 2005.

[40] A. Doshi, M. M. Trivedi, "Tactical Driver Behavior Prediction and Intent Inference: A Review," in Proc. 14 ${ }^{\text {th }}$ Int. IEEE Conf. on Int. Trans. Sys., Washington, DC, USA, pp. 1892-1897, 2011.

[41] Online]. Available: http://www.interactive-ip.eu 